\title{
Characterization of a Novel NDM-5-Harboring Plasmid from a Carbapenem-Resistant Escherichia coli Isolate from China
}

This article was published in the following Dove Press journal: Infection and Drug Resistance

\author{
Dongdong Yin ${ }^{1,2, *}$ \\ Yanfeng Lin ${ }^{1,3, *}$ \\ Zhonghong $\mathrm{Li}^{1,4, *}$ \\ Hui $\mathrm{Ma}^{5, *}$ \\ Lanfen Lu ${ }^{6}$ \\ Kaiying Wang ${ }^{1,3}$ \\ Lang Yang ${ }^{1,3}$ \\ Xinying Du' \\ Peng $\mathrm{Li}^{\prime}$ \\ Kezong $\mathrm{Qi}^{2}$ \\ Hongbin Song ${ }^{1} * *$
}

'Center for Disease Control and Prevention of PLA, Beijing, People's Republic of China;

${ }^{2}$ Anhui Province Key Laboratory of Veterinary Pathobiology and Disease

Control College of Animal Science and

Technology, Anhui Agricultural University, Hefei, People's Republic of China; ${ }^{3}$ Institute for Disease Control and Prevention, AMMS, Beijing, People's Republic of China; ${ }^{4}$ College of Environmental and Chemical Engineering, Nanchang Hangkong University, Nanchang, People's Republic of China; ${ }^{5}$ The Sixth Medical Center of PLA General Hospital, Beijing, People's Republic of China; ${ }^{6}$ Department of Laboratory Diagnosis, Sun Yat-Sen University Affiliated Zhongshan Hospital, Zhongshan, People's Republic of China

*These authors contributed equally to this work

Correspondence: Peng Li; Kezong Qi Email jiekenlee@I26.com;

qkz@ahau.edu.cn
Background: A carbapenem-resistant Escherichia coli (sequence type 5415) strain was isolated from a male patient through routine surveillance in 2018 in Guangzhou, China.

Materials and Methods: Bacteria were isolated from a sputum culture and identified by using the Vitek 2 compact system. The $b l a_{N D M-5}$ gene was amplified and confirmed by sequencing. Antimicrobial susceptibility testing was determined by a Vitek 2 compact system. The $b l a_{N D M-5}$ gene was located by Southern blotting. Whole-genome sequencing was carried out using both Illumina MiSeq and Oxford Nanopore MinION.

Results: S1-PFGE and Southern blotting showed that the bla $a_{\mathrm{NDM}-5}$ gene was located on a novel 66-kb IncFII [F2:A-:B-] plasmid. Conjugation assays revealed that the bla $_{\mathrm{NDM}-5^{-}}$ bearing plasmid was self-transferrable. Genomic sequencing and comparative analysis suggested that plasmid p2947-NDM5 likely originated from a combination of an IncFII-type backbone and the $b l a_{\mathrm{NDM}-5}$ flanking genetic elements.

Conclusion: This is the first report of an ST5414 E. coli strain expressing an NDM-5 $\beta-$ lactamase. This study highlights the genetic complexity of bla $a_{\mathrm{NDM}-5}$ carrying plasmids and the urgent need for continuous active monitoring.

Keywords: Escherichia coli, ST5415, NDM-5, IncFII, carbapenem resistant

\section{Introduction}

Carbapenemase-producing Enterobacteriaceae (CPE) constitute a public health problem in terms of both hospital- and community-acquired infections. ${ }^{1}$ Since the identification of NDM-1 in a Swedish traveler returning from India in $2009,{ }^{2}$ NDM enzymes have received special attention due to their rapid global spread and frequent association with other resistance genes. NDM-5 was first identified in an Escherichia coli strain isolated from a patient who had been hospitalized in India. ${ }^{3}$ Since then, NDM-5 has been detected in different countries around the world. ${ }^{4-8}$ The amino acid sequence of NDM- 5 differs from that of NDM- 1 at positions 88 $(\mathrm{Val} \rightarrow$ Leu) and 154 (Met $\rightarrow$ Leu), which confer a high level of hydrolytic activity against carbapenems. ${ }^{3}$ The rapid evolution and dissemination of NDM-5 represent a crucial challenge for clinical treatments.

Extraintestinal E. coli is a relatively common pathogen causing community and infections among Enterobacteriaceae in China. The acquisition of NDM is a great concern since it would greatly limit the treatments for E. coli that frequently carry multiple resistance determinants. Identifying clones or plasmids with $b l a_{\mathrm{NDM}}$ genes 
is important for understanding the epidemiology of resistance and controlling the spread of NDM in communities and healthcare systems. ${ }^{9,10}$

In this study, we report the emergence of the NDM5-producing E. coli strain ST5414 in China and characterized a novel plasmid carrying the $b l a_{\mathrm{NDM}-5}$ gene using Illumina and Nanopore sequencing platforms.

\section{Materials and Methods}

Identification of the E. coli Strain Carrying bla NDM

A carbapenem-resistant strain, ECO2947, was recovered from a sputum culture of a patient through routine surveillance in 2018 in Guangzhou, China. The species of strain ECO2947 was identified by the Vitek 2 compact system (bioMérieux, France). The $b l a_{\mathrm{NDM}}$ gene was detected by PCR and sequencing with primers $b l a_{\mathrm{NDM}^{-}}-\mathrm{F}$ (5'-GGC GGAATGGCTCATCACGA-3') and bla NDM $^{-}$R (5'-CG CAACACAGCCTG ACTTTC-3'). ${ }^{11,12}$ Ethics committee approval was obtained from the institutional review board of Sun Yat-Sen University Affiliated Zhongshan Hospital for these isolates, and verbal informed consent from patient was also accepted and approved by Sun YatSen University Affiliated Zhongshan Hospital. All experiments were conducted in accordance with relevant regulations and approved by the Chinese PLA Center for Disease Control and Prevention.

\section{SI-PFGE, Southern Blotting and Conjugation}

Bacterial genomic DNA from strain ECO2947 was prepared in agarose plugs and digested with the S1 endonuclease (Takara, Dalian, China). DNA fragments were separated by pulsed-field gel electrophoresis (PFGE) through a CHEF-DR III system (Bio-Rad, Hercules, USA). The conditions of the PFGE run were $6.0 \mathrm{~V} / \mathrm{cm}$ gradient, $120^{\circ}$ angle, and 7- to 26-second pulse times for $15 \mathrm{~h}$. The plasmid DNA was transferred to a positively charged nylon membrane (Solabio, China) and hybridized with the digoxigenin-labeled specific probe to $b l a_{\mathrm{NDM}-5}$. The experiment was performed according to the manufacturer's manual of the DIG High Prime DNA Labeling and Detection Start Kit I (Cat. No: 11,745,832,910, Roche).

Conjugation experiments were performed by broth and filter mating using strain ECO2947 as the donor and azideresistant E. coli J53 as the recipient. Strains ECO2947 and J53 were mixed (ratio of 1:3) in Luria-Bertani (LB) broth, which was used to make LB agar plates, and incubated for $18 \mathrm{~h}$. The mixture was spread on a selective MacConkey agar plate containing meropenem $(4 \mu \mathrm{g} / \mathrm{mL})$ and sodium azide $(150 \mu \mathrm{g} / \mathrm{mL})$ to select transconjugants. Horizontal transferability of drug resistance was evaluated by antimicrobial susceptibility testing, and the corresponding transconjugants were confirmed by S1-PFGE.

\section{Antimicrobial Susceptibility Testing}

The minimal inhibitory concentrations (MICs) of amikacin, ampicillin, sulbactam/ampicillin, aztreonam, furadantin, ciprofloxacin, piperacillin/tazobactam, gentamicin, cefepime, ceftriaxone, ceftazidime, cefotetan, cefamedin, tobramycin, imipenem and levofloxacin were determined by a Vitek 2 compact system (bioMérieux, France) following the manufacturer's instructions. The results were interpreted following the guidelines of the Clinical and Laboratory Standards Institute (CLSI) ${ }^{13}$

\section{Whole Sequencing and Analysis}

Genomic DNA was extracted using a High Pure PCR Template Preparation Kit (Roche, Basel, Switzerland). Sequencing was carried out using both Illumina MiSeq and Oxford Nanopore MinION. The de novo hybrid assembly of short Illumina reads and long MinION reads was performed using Unicycler v0.4. $8^{14}$ with the conservative mode. Complete circular contigs were corrected using Pilon with Illumina reads for several rounds until no change was detected. Genome sequences were annotated using the RAST server. ${ }^{15}$ The sequence type was determined through the MLST web server. ${ }^{16}$ Virulence genes and plasmid types were identified using VirulenceFinder, PlasmidFinder and pMLST. ${ }^{17}$

\section{Nucleotide Sequence Accession Number}

The complete sequences of the chromosome of strain ECO2947, plasmid p2947-D and p2947-NDM5 have been deposited in GenBank under accession numbers CP046259, CP046260 and CP046261, respectively.

\section{Results}

\section{Bacterial Identification and Susceptibility Testing}

Strain ECO2947 was identified as E. coli using the Vitek 2 compact system and confirmed by 16S rRNA sequencing. The MIC values of the tested antimicrobials revealed that E. coli ECO2947 exhibited resistance to nearly all tested 
$\beta$-lactam antibiotics, including ampicillin, sulbactam/ampicillin, piperacillin/tazobactam, ceftriaxone, ceftazidime, cefepime, cefamedin and imipenem, with the exception of aztreonam (Table 1). PCR amplification and sequencing confirmed the presence of $b l a_{\mathrm{NDM}-5}$.

\section{Microbiological and Genomic Features of E. coli ECO2947}

S1 PFGE showed that E. coli ECO2947 contained two different plasmids ( $\sim 66 \mathrm{~kb}$ and $\sim 108 \mathrm{~kb}$ ) (Figure 1). Southern blotting revealed that the $b l a_{\mathrm{NDM}-5}$ gene was located on the $\sim 66 \mathrm{~kb}$ plasmid (named p2947-NDM5), which was transferred to $E$. coli $\mathrm{J} 53$ at a frequency of $1.63 \times 10^{-2}$ transconjugants per donor cell.

The transconjugants acquired resistance to ampicillin, ceftriaxone, sulbactam/ampicillin, piperacillin/tazobactam, ceftazidime, cefamedin, and imipenem (Table 1), and the MIC values of carbapenems in the transconjugants were considerably increased compared with those of the recipient strain E. coli J53.

E. coli $\mathrm{ECO} 2947$ was further subjected to sequencing using both MiSeq and MinION sequencing. Genomic analysis revealed that strain E. coli $\mathrm{ECO} 2947$ belonged to a novel sequence type ST5414 and had a 4,884,967 bp chromosome and two plasmids. Twelve virulence factors were found in the genome: single copies of eae (intimin), espA (type III secretion system), espB (secreted protein B), espF (type III secretion system), iss (increased serum survival), lpfA (long polar fimbriae), nleA (non-LEE encoded effector A), nleB (nonLEE encoded effector B), nleC (non-LEE encoded effector C), tir (translocated intimin receptor protein) and two copies of gad (glutamate decarboxylase). A screening for acquired resistance determinants found that the chromosome only possessed the resistance gene $m d f(A)$ (prototypic secondary multidrug transporter), while the plasmid p2947-NDM5 carried only $b l a_{\mathrm{NDM}-5}$, and the other plasmid (named p2947-D) carried multiple resistance genes, including sul2 (sulfonamide resistance), qnrS1 (fluoroquinolone resistance), aph (3")-Ib and $a p h(6)$-Id (aminoglycoside resistance).

\section{Characterization of the Novel blaNDM-5-Harboring Plasmid}

The $b l a_{\mathrm{NDM}-5}$-harboring plasmid $\mathrm{p} 2947-\mathrm{NDM} 5$ belonged to the incompatibility type IncFII [F2:A-:B-] with a length of $66,053 \mathrm{bp}$, an average $\mathrm{G}+\mathrm{C}$ content of $52.41 \%$ and 94 predicted coding sequences. p2947-NDM5 had a 61-kb backbone and a 5-kb multidrug resistance (MDR) region. A BLAST search revealed that p2947-NDM5 was highly similar to plasmid p974-NDM of E. coli strain 974 (accession number: MG825370.1) (99\% coverage and 99.66\% identity), plasmid unnamed4 of Klebsiella pneumoniae strain 4743 (accession number: CP033629.1) (93\% coverage and 100\% identity, referred to as p4743) and the plasmid of Salmonella enterica subsp. enterica serovar Derby strain 75 (accession number: MK191836.1) (92\% coverage and 99.97\% identity, referred to as p75). The four plasmids have almost identical

Table I Antibiotic Susceptibilities of E. coli ECO2947 and the E. coli J53 Transconjugants

\begin{tabular}{|l|l|l|l|}
\hline \multirow{2}{*}{ Antimicrobial } & \multicolumn{2}{l|}{ MIC $(\boldsymbol{\mu g} / \mathbf{m L})$} & \\
\cline { 2 - 4 } & ECO2947 & J53 (The Transconjugant) & J53 \\
\hline Ampicillin & $\geq 32$ & $\geq 32$ & 8 \\
Sulbactam-Ampicillin & $\geq 32$ & $\geq 32$ & 4 \\
Aztreonam & $\leq 1$ & $\leq 1$ & $\leq 1$ \\
Furadantin & 32 & $\leq 16$ & $\leq 16$ \\
Ciprofloxacin & $\leq 0.25$ & $\leq 0.25$ & $\leq 0.25$ \\
Piperacillin-Tazobactam & 64 & 64 & $\leq 4$ \\
Gentamicin & $\leq 1$ & $\leq 1$ & $\leq 1$ \\
Cefepime & 16 & 8 & $\leq 1$ \\
Ceftriaxone & $\geq 64$ & $\geq 64$ & $\leq 1$ \\
Ceftazidime & $\geq 64$ & $\geq 64$ & $\leq 1$ \\
Cefotetan & 32 & 32 & $\leq 4$ \\
Cefamedin & $\geq 64$ & $\geq 64$ & $\leq 4$ \\
Tobramycin & $\leq 1$ & $\leq 1$ & $\leq 1$ \\
Imipenem & $\geq 16$ & $\geq 16$ & $\leq 1$ \\
Amikacin & $\leq 2$ & $\leq 2$ & $\leq 2$ \\
Levofloxacin & $\mathrm{I}$ & $\leq 0.25$ & $\leq 0.25$ \\
\hline
\end{tabular}




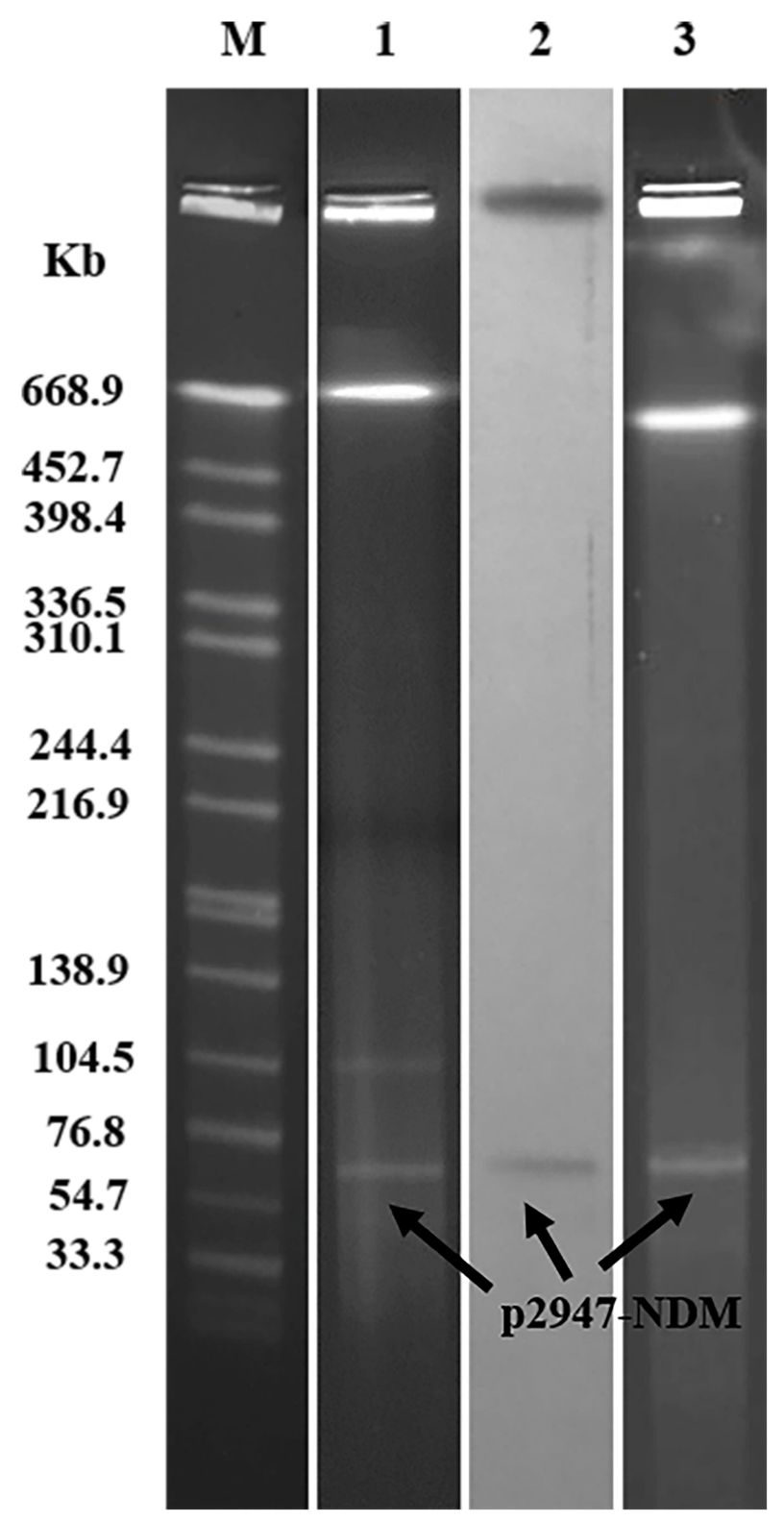

Figure I SI-PFGE pattern for strain ECO2947 and southern blotting for the bla $a_{\text {NDM-5 }}$ gene. Lanes: Marker, Salmonella serotype Braenderup strain $\mathrm{H} 9812$ as the size standard; I, PFGE result for SI-digested plasmid DNA of strain ECO2947; 2, Southern blotting with the probe specific to bla $a_{\text {NDM-5 }} ; 3$, PFGE patterns for SI-digested plasmid DNA of $E$. coli transconjugants J53.

backbones and contain a set of core genes responsible for plasmid replication (repA), conjugation/T4SS (tra and trb genes), stability ( $s t d B)$ and segregation (parM) (Figure 2A). However, p2947-NDM5 had quite different MDR regions from these similar plasmids. In p2947-NDM5, $b a_{\mathrm{NDM}-5}$

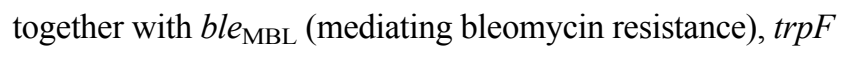
(encoding the phosphoribosylanthranilate isomerase), tat (encoding tat twin-arginine translocation pathway signal sequence domain protein) and $c t u A l$ (encoding periplasmic divalent cation tolerance protein) was bracketed by IS5 and
IS26, while p974-NDM had the bla $a_{\mathrm{NDM}-1}$ gene instead and an additional region composed of IS26, ISKox3, resolvase, Tn2 transposon, IS 3000 and $\triangle \mathrm{IS} A$ ba 125. Plasmids $\mathrm{p} 75$ and p4743 had quite different resistance genes and genetic contexts. Both p75 and p4743 had two copies of IS26 at each end; the former was composed of IS26-IS903-BtuB-tnpR Tn3-IS26, while the latter was composed of IS26-intl1IS26- $\Delta b l a_{\mathrm{TEM}^{-}}-W b u C$-bla $a_{\mathrm{CTX}-\mathrm{M}-15}$-IS26 (Figure 2B).

Comparative analysis revealed that the genetic context of $b l a_{\mathrm{NDM}-5}(\sim 5100 \mathrm{bp})$ in p2947-NDM5 was nearly identical to those previously reported in pGZ3-NDM5 (accession number: CP017981.1) (100\% coverage and 100\% identity), pNDM-HK3774 (accession number: MI1234502.1) (100\% coverage and 100\% identity), pHNAH699 (accession number: MH286952.1) (100\% coverage and 100\% identity) and pP855-NDM5 (accession number: MF547508.1) (100\% coverage and $99.98 \%$ identity) (Figure 2C). However, compared with the above reference plasmids, p2947-NDM5 lacked IS3000.

\section{Discussion}

Due to the intrinsic and acquired resistance of E. coli, this bacterium constitutes a serious clinical threat that limits the choice of treatment. Most reports have indicated a high ST diversity for $b l a_{\mathrm{NDM}-5}$-positive E. coli. ${ }^{4,18,19}$ The MLST analysis revealed that E. coli ECO2947 belongs to ST5414, which is unlike the ST types of NDM-5-producing E. coli ST167 (China), ${ }^{18}$ ST540 (Japan), ${ }^{20}$ ST648 (Australia), ST648 (UK) ${ }^{3}$ and ST648 (India). This appears to be the first report of an ST5414 E. coli strain expressing an NDM$5 \beta$-lactamase. This is a worrying development, as it demonstrates the further spread of $b l a_{\mathrm{NDM}-5}$ among different ST types of E. coli, and the transfer frequency of p2947-NDM5 demonstrated its great potential to transfer across species.

IncFII-type plasmids are a group of plasmid families with similar replicons and transfer regions, ${ }^{21}$ which are spread among Enterobacteriaceae in humans and animals worldwide. ${ }^{6,22,23}$ Although the IncFII-type plasmids are narrow host plasmids, the plasmid can adapt well to $E$. coli, are easy to conjugate and transfer, and facilitates the spread of resistance genes. ${ }^{24-26}$ Horizontal gene transfer also promotes the widespread dissemination of $b l a_{\mathrm{NDM}}$ in Enterobacterales. ${ }^{27}$ In this study, two of the three plasmids highly similar to p2947-NDM5 were from animals. Klebsiella pneumoniae strain 4743 was isolated from humans in Italy, the Salmonella enterica subsp. enterica serovar Derby strain was isolated from swine in the United States, and the E. coli strain 974 was isolated from a pig in 
A

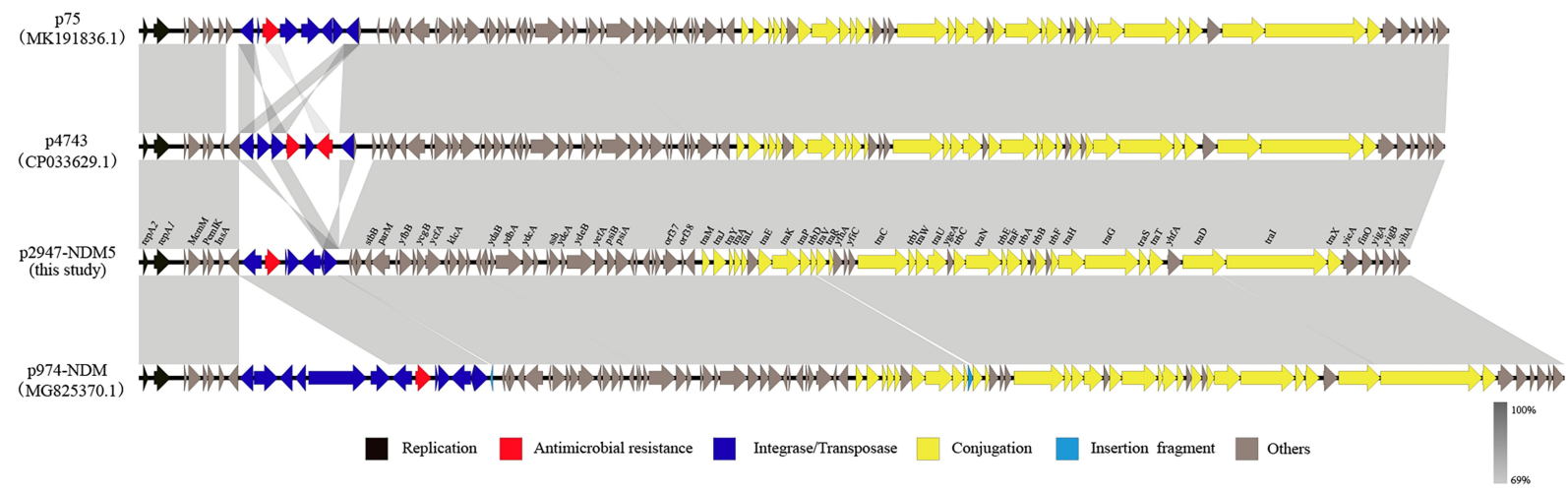

B

$\mathrm{p} 75$
$(\mathrm{CP} 033629.1)$

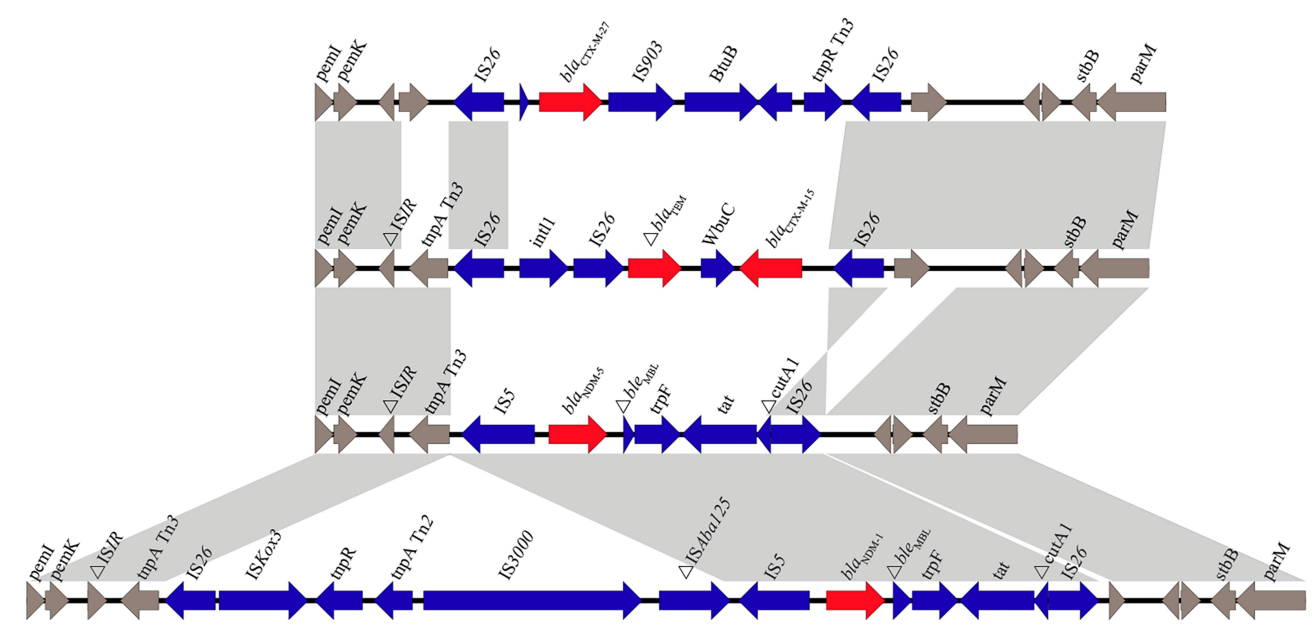

p4743
(MK191836.1)

p974-NDM (MG825370.1)

p2947-NDM5
(this study)

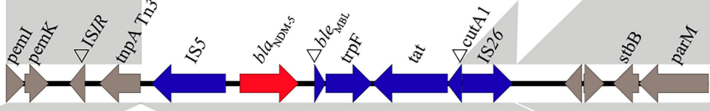

C

p2947-NDM5
this study
pGZ3 NDM5
CP017981.1
pNDM-HK3774
MH234502.1
pP855-NDM5
MF547508.1
pHNAH699
MH286952.1
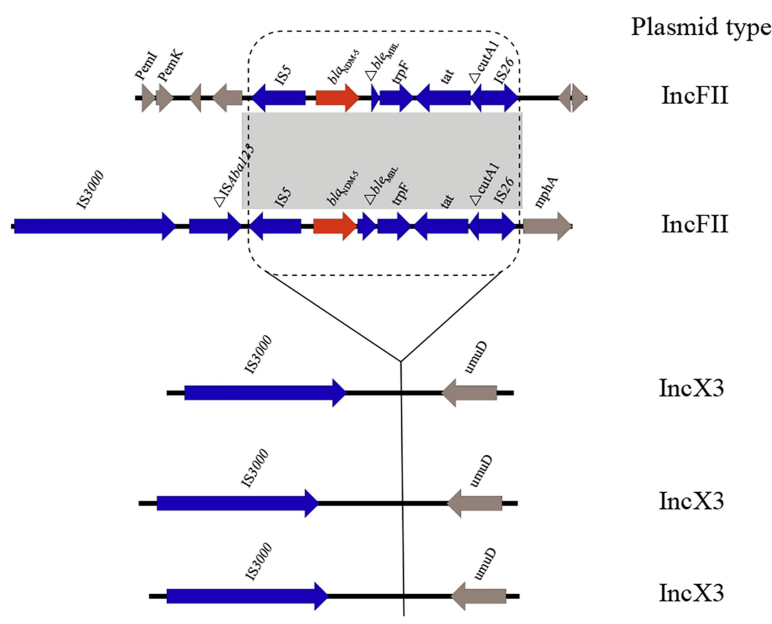

Size

Area

Isolate Year

$66,053 \mathrm{bp}$

Guangzhou

human

2018

91,451 bp

Guangzhou human

2015

Figure 2 Plasmid analysis of p2947-NDM5. (A) Genetic structure comparison of p2947-NDM5, CP033629.I), MK 191836.1) and MG825370.I). (B) Comparative analysis of MDR regions. (C) Comparative analysis of the genetic contexts of bla NDM-5 in plasmids reported in this study and previously described. 
Hong Kong. Additionally, the genetic context of $b l a_{\mathrm{NDM}-5}$ of p2947-NDM5 has also been found on different types of plasmids and in different species, and a conjugation assay revealed that p2947-NDM5 was self-transferrable. From this, we speculated that p2947-NDM5 was the genetic context of $b l a_{\mathrm{NDM}-5}$, which had been inserted into the IncFII [F2:A-:B-] plasmid backbone during transmission, and p2947-NDM5 has a potential risk of spread across species. Therefore, the association of IncFII plasmids and $b l a_{\mathrm{NDM}}$ variants and the epidemiology of IncFII plasmids in Enterobacteriaceae warrant more studies.

\section{Conclusion}

In summary, we identified a $b l a_{\mathrm{NDM}-5}$-positive $E$. coli strain, ST5414, for the first time. The $b l a_{\mathrm{NDM}-5}$ gene was located on a novel self-transferrable IncFII-type plasmid. Our study highlights the potential spread of carbapenemresistant plasmids among Enterobacteriaceae. Further research is necessary to take urgent and effective surveillance measures and to control the spread of the $b l a_{\mathrm{NDM}-5^{-}}$ carrying IncFII plasmids.

\section{Author Contributions}

All authors made substantial contributions to conception and design, acquisition of data, or analysis and interpretation of data; took part in drafting the article or revising it critically for content; gave final approval of the version to be published and agreed to be accountable for all aspects of the work.

\section{Funding}

The study was supported by grants from the National Science and Technology Major Project (no. 2018ZX10201001-003 and no. 2018ZX10712001-002-002), the Beijing Natural Science Foundation (no. 5172029) and the Beijing Nova Program (no. Z181100006218110).

\section{Disclosure}

The authors report no conflicts of interest in this work.

\section{References}

1. Nordmann P, Dortet L, Poirel L. Carbapenem resistance in Enterobacteriaceae: here is the storm! Trends Mol Med. 2012;18 (5):263-272. doi:10.1016/j.molmed.2012.03.003

2. Yong D, Toleman MA, Giske CG, et al. Characterization of a new metallo-beta-lactamase gene, $b l a_{\mathrm{NDM}-1}$, and a novel erythromycin esterase gene carried on a unique genetic structure in Klebsiella pneumoniae sequence type 14 from India. Antimicrob Agents Chemother. 2009;53(12):5046-5054. doi:10.1128/AAC.00774-09
3. Hornsey M, Phee L, Wareham DW. A novel variant, NDM-5, of the New Delhi metallo- $\beta$-lactamase in a multidrug-resistant Escherichia coli ST648 isolate recovered from a patient in the United Kingdom. Antimicrob Agents Chemother. 2011;55(12):5952-5954. doi:10.1128/ AAC.05108-11

4. Sassi A, Loucif L, Gupta SK, et al. NDM-5 carbapenemase-encoding gene in multidrug-resistant clinical isolates of Escherichia coli from Algeria. Antimicrob Agents Chemother. 2014;58(9):5606-5608. doi:10.1128/AAC.02818-13

5. Nakano R, Nakano A, Hikosaka K, et al. First report of metallo- $\beta$ lactamase NDM-5-producing Escherichia coli in Japan. Antimicrob Agents Chemother. 2014;58(12):7611-7612. doi:10.1128/AAC.04265-14

6. Pitart C, Sole M, Roca I, et al. Molecular characterization of blaNDM-5 carried on an IncFII plasmid in an Escherichia coli isolate from a nontraveler patient in Spain. Antimicrob Agents Chemother. 2015;59(1):659-662. doi:10.1128/AAC.04040-14

7. de Man TJ, Perry KA, Avillan JJ, et al. Draft genome sequence of a New Delhi metallo- $\beta$-lactamase-5 (NDM-5)-producing multidrugresistant Escherichia coli isolate. Genome Announc. 2015;3(2). doi:10.1128/genomeA.00017-15

8. Wailan AM, Paterson DL, Caffery M, et al. Draft genome sequence of NDM-5-producing Escherichia coli sequence type 648 and genetic context of blaNDM-5 in Australia. Genome Announc. 2015;3(2). doi:10.1128/genomeA.00194-15

9. Giufre M, Errico G, Accogli M, et al. Emergence of NDM-5-producing Escherichia coli sequence type 167 clone in Italy. Int J Antimicrob Agents. 2018;52(1):76-81. doi:10.1016/j. ijantimicag.2018.02.020

10. Dortet L, Poirel L, Nordmann P. Worldwide dissemination of the NDM-type carbapenemases in gram-negative bacteria. Biomed Res Int. 2014;2014:249856. doi:10.1155/2014/249856

11. Frank JA, Reich CI, Sharma S, et al. Critical evaluation of two primers commonly used for amplification of bacterial 16S rRNA genes. Appl Environ Microbiol. 2008;74:2461-2470. doi:10.1128/ AEM.02272-07

12. Zong Z, Zhang X. bla NDM-1 -carrying acinetobacter johnsonii detected in hospital sewage. J Antimicrob Chemother. 2013;68(5):1007-1010. doi:10.1093/jac/dks505

13. Clinical and Laboratory Standards Institute. Performance Standards for Antimicrobial Susceptibility Testing: Twenty-Eighth Informational Supplement. M100-S28. Wayne, PA, USA: CLSI; 2018.

14. Wick RR, Judd LM, Gorrie CL, et al. Unicycler: resolving bacterial genome assemblies from short and long sequencing reads. PLoS Comput Biol. 2017;13(6):e1005595. doi:10.1371/journal.pcbi.100 5595

15. Aziz RK, Bartels D, Best AA, et al. The RAST server: rapid annotations using subsystems technology. BMC Genom. 2008;9(1):75. doi:10.1186/1471-2164-9-75

16. Larsen MV, Cosentino S, Rasmussen S, et al. Multilocus sequence typing of total-genome-sequenced bacteria. J Clin Microbiol. 2012;50 (4):1355-1361. doi:10.1128/JCM.06094-11

17. Carattoli A, Zankari E, Garcia-Fernandez A, et al. In silico detection and typing of plasmids using PlasmidFinder and plasmid multilocus sequence typing. Antimicrob Agents Chemother. 2014;58:3895-3903. doi:10.1128/AAC.02412-14

18. Yang P, Xie Y, Feng P, et al. blaNDM-5 carried by an IncX3 plasmid in Escherichia coli sequence type 167. Antimicrob Agents Chemother. 2014;58(12):7548-7552. doi:10.1128/AAC.03911-14

19. Zhang F, Xie L, Wang X, et al. Further spread of $b l a_{\mathrm{NDM}-5}$ in Enterobacteriaceae via IncX3 plasmids in Shanghai, China. Front Microbiol. 2016;7:424. doi:10.3389/fmicb.2016.00424

20. Nordmann P. Carbapenemase-producing Enterobacteriaceae: overview of a major public health challenge. Med Mal Infect. 2014;44 (2):51-56. doi:10.1016/j.medmal.2013.11.007 
21. Laura V, Aurora G-F, Daniela F, et al. Replicon sequence typing of IncF plasmids carrying virulence and resistance determinants. $J$ Antimicrob Chemother. 2010:65(12):2518-2529.

22. Chen L, Chavda KD, Melano RG, et al. Complete sequence of a blaKPC-2 -harboring IncFIIK1 plasmid from a Klebsiella pneumoniae sequence type 258 strain. Antimicrob Agents Chemother. 2013;57(3):1542-1545. doi:10.1128/AAC.02332-12

23. Cheng VC, Wong SC, Ho PL, et al. Strategic measures for the control of surging antimicrobial resistance in Hong Kong and mainland of China. Emerg Microbes Infect. 2015;4(1):e8. doi:10.1038/emi.2015.8

24. Ho PL, Chan J, Lo WU, et al. Dissemination of plasmid-mediated fosfomycin resistance fosA3 among multidrug-resistant Escherichia coli from livestock and other animals. J Appl Microbiol. 2013;114 (3):695-702. doi:10.1111/jam.12099
25. Lo WU, Chow KH, Law PY, et al. Highly conjugative IncX4 plasmids carrying bla CTX-M in Escherichia coli from humans and food animals. J Med Microbiol. 2014;63(6):835-840. doi:10.1099/ jmm.0.074021-0

26. Ho PL, Lo WU, Yeung MK, et al. Dissemination of pHK01-like incompatibility group IncFII plasmids encoding CTX-M-14 in Escherichia coli from human and animal sources. Vet Microbiol. 2012;158(1-2):172-179. doi:10.1016/j.vetmic.2012.02.004

27. Guo X, Rao Y, Guo L, et al. Detection and genomic characterization of a Morganella morganii isolate from China that produces NDM-5. Front Microbiol. 2019;10:1156. doi:10.3389/fmicb.2019.01156

\section{Publish your work in this journal}

Infection and Drug Resistance is an international, peer-reviewed openaccess journal that focuses on the optimal treatment of infection (bacterial, fungal and viral) and the development and institution of preventive strategies to minimize the development and spread of resistance. The journal is specifically concerned with the epidemiology of antibiotic resistance and the mechanisms of resistance development and diffusion in both hospitals and the community. The manuscript management system is completely online and includes a very quick and fair peerreview system, which is all easy to use. Visit http://www.dovepress.com/ testimonials.php to read real quotes from published authors. 